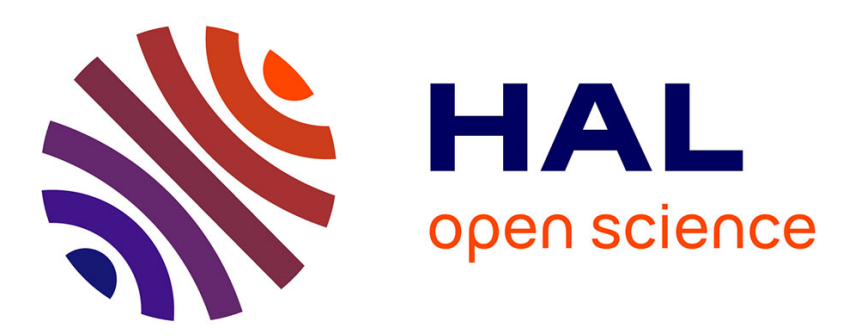

\title{
Strongly enhanced laser absorption and electron acceleration via resonant excitation of surface plasma waves
}

\author{
M. Raynaud, J. Kupersztych, C. Riconda, J. C. Adam, A. Heron
}

\section{To cite this version:}

M. Raynaud, J. Kupersztych, C. Riconda, J. C. Adam, A. Heron. Strongly enhanced laser absorption and electron acceleration via resonant excitation of surface plasma waves. Physics of Plasmas, 2007, 14 (9), pp.092702. 10.1063/1.2755969 . hal-01327434

\section{HAL Id: hal-01327434 \\ https://hal.science/hal-01327434}

Submitted on 8 Jun 2016

HAL is a multi-disciplinary open access archive for the deposit and dissemination of scientific research documents, whether they are published or not. The documents may come from teaching and research institutions in France or abroad, or from public or private research centers.
L'archive ouverte pluridisciplinaire HAL, est destinée au dépôt et à la diffusion de documents scientifiques de niveau recherche, publiés ou non, émanant des établissements d'enseignement et de recherche français ou étrangers, des laboratoires publics ou privés. 


\title{
Strongly enhanced laser absorption and electron acceleration via resonant excitation of surface plasma waves
}

\author{
M. Raynaud ${ }^{\text {a) }}$ and J. Kupersztych \\ DSM/DRECAM/SPCSI, CEA/Saclay, 91191 Gif-sur-Yvette Cedex, France \\ C. Riconda \\ PAPD/LULI, Université Paris 6, CNRS, CEA, Ecole Polytechnique, 3, rue Galilée, 94200 Ivry-sur-Seine, \\ France and CELIA, Université Bordeaux 1, 33405 Talence Cedex, France
}

J. C. Adam and A. Héron

CPhT, Ecole Polytechnique, 91128 Palaiseau Cedex, France

(Received 27 October 2006; accepted 14 June 2007; published online 20 September 2007)

\begin{abstract}
Two-dimensional (2D) particle-in-cell numerical simulations of the interaction between a high-intensity short-pulse $p$-polarized laser beam and an overdense plasma are presented. It is shown that, under appropriate physical conditions, a surface plasma wave can be resonantly excited by a short-pulse laser wave, leading to strong relativistic electron acceleration together with a dramatic increase, up to $70 \%$, of light absorption by the plasma. Purely $2 \mathrm{D}$ effects contribute to enhancement of electron acceleration. It is also found that the angular distribution of the hot electrons is drastically affected by the surface wave. The subsequent ion dynamics is shown to be significantly modified by the surface plasma wave excitation. (c) 2007 American Institute of Physics.
\end{abstract}

[DOI: 10.1063/1.2755969]

\section{INTRODUCTION}

Fast electrons generated in the interaction of intense ultrashort laser pulses with solid targets play an important role for numerous applications such as the development of intense sources of high-energy particles ${ }^{1}$ or photons ${ }^{2}$ and in the fast ignitor scheme in the framework of inertial fusion. ${ }^{3}$ In this context, particular attention has been focused on the possibility of creating, among various electron acceleration schemes, ${ }^{4,5}$ large amplitude plasma waves in the wake of a laser pulse. ${ }^{6}$ Recently, much attention has been raised to laser absorption mechanisms and high-energy electron generation involving overdense plasmas with stepwise density profiles resulting from laser-solid interaction. ${ }^{7-9}$

Intense ultrashort (say $100 \mathrm{fs}$ ) laser pulses impinging onto a solid target rapidly induce multiple ionization without significant ablation while the hydrodynamic expansion, which is partially reduced by the radiation pressure of the laser, has not enough time to smooth the density gradient. An overdense plasma can thus be created which can keep the steep density profile of the original target. Electromagnetic energy is then absorbed in an optical skin depth by collisional processes and through collisionless mechanisms such as sheath inverse bremsstrahlung, ${ }^{10} \vec{J} \times \vec{B}$ heating, ${ }^{11}$ vacuum heating, ${ }^{12}$ and anomalous skin-layer heating, ${ }^{13}$ while the electron thermal energy is correlatively transported into the dense cold plasma.

Conversion of the laser energy into electron energy usually does not exceed a few tens of percents in an overdense plasma with step-like density profile obtained in ultrashort high-intensity interaction regime. ${ }^{14}$ To improve absorption of

\footnotetext{
${ }^{a)}$ Present address: DSM/DRECAM/LSI, CEA/Saclay, 91191 Gif-sur-Yvette Cedex, France.
}

the laser energy, advantage can be taken of surface plasma wave excitation. ${ }^{15-20}$ Under certain conditions, these waves can be supported by an overdense plasma with a stepwise density profile. ${ }^{21}$ Such laser-excited surface plasma waves are localized at the plasma-vacuum interface. They are characterized by a large resonant electric field mainly directed perpendicularly to the surface of the plasma and with a small spatial extension. This strongly inhomogeneous field can then accelerate electrons toward the vacuum and inside the plasma via ponderomotive acceleration more efficiently than the laser. It is interesting to note that, in the framework of multiphoton photoelectric emission of metals, laser excitation of surface waves can considerably modify the energy of the photoemitted electrons, leading to an increase of the laser absorption together with a strong enhancement of the electron yield. ${ }^{22,23}$ In this context, energetic photoelectrons have been produced with energies up to $2 \mathrm{keV}$ for laser intensities in the range $10^{10}-10^{13} \mathrm{~W} / \mathrm{cm}^{2} .{ }^{24}$ It has been experimentally demonstrated that the origin of the electron energy gain is the ponderomotive acceleration of the emitted electrons by the field of the surface waves.

In a first exploratory study, ${ }^{19}$ we had investigated the relativistic one-dimensional motion of test electrons in an external electric field modelling that of a surface wave excited by ultrashort laser pulses. It was shown that quasimonokinetic relativistic electron bunches with energies of several $\mathrm{MeV}$ can be produced with laser intensities up to $10^{18} \mathrm{~W} / \mathrm{cm}^{2}$. However, the possibility of creating a surface plasma wave by the ultrashort high-intensity laser pulses could not be investigated. Also, the effect of the space charge field which grows with the interaction duration could not be taken into account while it is expected to play an important part in the interaction. Actually, the space charge field recoils back the electron bunches in the plasma, leading to a true 
conversion process of the surface wave energy in a sort of "enhanced vacuum heating" in the field of the surface wave. The aim of the present paper is thus to show the possibility, in the ultrashort laser pulse regime, of creating a selfconsistent surface plasma wave in a sharp-edged overdense plasma in order to fully investigate the mechanism of relativistic electron acceleration by the field of the plasma wave. The laser-plasma interaction is investigated by performing 2D particle-in-cell (PIC) numerical simulations. A comparison between simulations involving the excitation of a surface plasma wave and simulations with the same physical parameters but without surface wave excitation has been systematically carried out. Our results will point out the great interest of resonant surface wave excitation to dramatically increase the light absorption by the plasma together with strong electron acceleration. Also, purely 2D effects are shown to contribute to a further enhancement of the maximum electron acceleration.

After describing the numerical model and setup in the first section of the paper, we shall describe the conditions for optimum surface plasma wave excitation. Next, we shall present the structure of the resonant field of the surface wave as obtained from the simulations. Electron acceleration and enhanced absorption of the laser energy in the plasma will then be discussed. We shall begin the study by focusing on short time scales comparable to the laser pulse duration. We shall then examine the subsequent evolution of the plasma over longer time scales in order to explore the novel question of ion acceleration through laser excitation of surface plasma waves.

\section{DESCRIPTION OF THE PHYSICAL SYSTEM}

In order to study the dynamics of the surface plasma wave excitation by a short laser pulse, we have performed numerical PIC simulations by means of the relativistic 2D PIC code EMI2D. ${ }^{25}$ An overdense plasma is considered with a steep density gradient directed along the $x$-direction of the $(x, y)$ simulation plane such that $n_{e} / n_{c}=25$, where $n_{e}$ and $n_{c}$ denote, respectively, the electron density and plasma critical density. The plasma is initially in thermal equilibrium with a Maxwellian distribution. The electron temperature is $T_{e}$ $=1 \mathrm{keV}$ with $T_{e} / T_{i}=10$, where $T_{i}$ denotes the ion temperature (here $m_{i} / m_{e}=1836$, where $m_{e}$ and $m_{i}$ are the electron and ion mass). The incoming laser beam is linearly $p$-polarized so that the laser electric field is in the $(x, y)$ simulation plane. This polarization is the only one possible for surface plasma wave excitation. The laser (of angular frequency $\omega_{0}$ and wavelength $\lambda_{0}$ ) is launched from the left boundary with a peak intensity of $I_{0} \lambda_{0}^{2}=10^{18} \mathrm{~W} \mu \mathrm{m}^{2} / \mathrm{cm}^{2}$ and is characterized by a temporal Gaussian shape and a pulse duration of $60 \mathrm{fs}$ (full width at half maximum). During the laser propagation in the vacuum, the ions are kept immobile. They are allowed to move as soon as the laser wave is impinging (with an angle $\vartheta$ ) onto the plasma surface.

The energy and momentum conservation equations in the "photon-plasmon (plasma wave)" scattering can be written as $\omega_{s w}=\omega_{0}$ and $k_{s w, y}=k_{0 y}$, where $\omega_{s w}$ and $k_{s w, y}$, respectively, denote the frequency and wave vector of the surface plasma wave directed along the $y$-direction of the plasmavacuum interface and $k_{0 y}$ denote the $y$-component of the wave vector. As the phase velocity of a surface plasma wave is lower than the light velocity, an additional constraint is required by momentum conservation for resonant excitation of such a wave. ${ }^{26}$ Thus, to satisfy the conditions for excitation of the surface plasma wave, we have considered the plasma surface to have a periodic shape along the $y$-direction keeping the density jump sharp across the surface. This periodic structure provides the increment to the light wave momentum to reach the momentum value of the surface wave, as a consequence of Bloch's theorem. ${ }^{26}$ The component $k_{0 y}^{\prime}$ of the photon wave vector directed along the direction of the periodic plasma surface expresses $k_{0 y}^{\prime}=\left(\omega_{0} / c\right) \quad(\sin \vartheta$ $+\lambda_{0} / a$ ), where $a$ denotes the surface density period. It is instructive to mention that if we consider the dispersion relation of the surface plasma waves, ${ }^{15}$ the resonance conditions $\left(\omega_{s w}=\omega_{0}\right.$ and $\left.k_{s w, y}=k_{0 y}^{\prime}\right)$ take the simple following form:

$$
k_{s w, y}=k_{0} \sqrt{\left(1-n_{e} / n_{c}\right) /\left(2-n_{e} / n_{c}\right)},
$$

where to a first approximation, the plasma is described as an electron fluid neutralized by an uniform ion background and we assume that the thermal corrections are negligible in this range of temperatures. ${ }^{15}$ As $n_{e} / n_{c}=25$, we have $k_{s w, y}$ $=(24 / 23)^{1 / 2} k_{0}$ and $\lambda_{s w, y} \sim \lambda_{0}$.

We choose the surface plasma modulation of sinusoidal shape with a periodicity parameter $a$ determined by the resonance conditions. For the given value of $n_{e} / n_{c}$ and for an angle of incidence of $\sim 30^{\circ}, a$ is of the order of $\lambda_{0}$. Various depths of the surface density modulation, from $\lambda_{0} / 2$ to $3 \lambda_{0} / 2$, have been tested. In this range of values, this parameter does not significantly affect the coupling with the laser nor the amplitude of the surface plasma wave. Further increase of the depth of the modulation induces shadows which disturb the surface plasma wave excitation. As a consequence, beyond a maximum depth of about $2 \lambda_{0}$, lower field enhancement and lesser light absorption are observed with respect to the value given hereafter.

In the following, we have taken the depth of the surface modulation equal to $\lambda_{0} / 2$, which allows avoiding shadow problems at the chosen angle of incidence of the laser wave. The box size along the $y$-direction is equal to $6 \lambda_{0}$ for $\vartheta$ $\sim 30^{\circ}$. Thus, when the resonance conditions are satisfied, we should have six wavelengths of the surface plasma wave along the $y$-direction. Comparisons between the case where a surface plasma wave is excited (resonant case) versus that without any surface plasma wave excitation (nonresonant case) will be done hereafter by simply taking a flat surface. However, test simulations performed with a modulated but nonresonant surface (to wit, incident angle of the laser beam not matching the above resonance conditions) have shown that additional electron heating and laser absorption can be ruled out, in the range of laser intensity and in the time scale regime considered here, as due to a purely geometrical effect. The vacuum length in front of the plasma in the $x$-direction is taken as $40 \lambda_{0}$ in order to allow spatial expansion of the particles and to minimize edge effects on the fast produced electrons. The initial spatial extent of the plasma is 

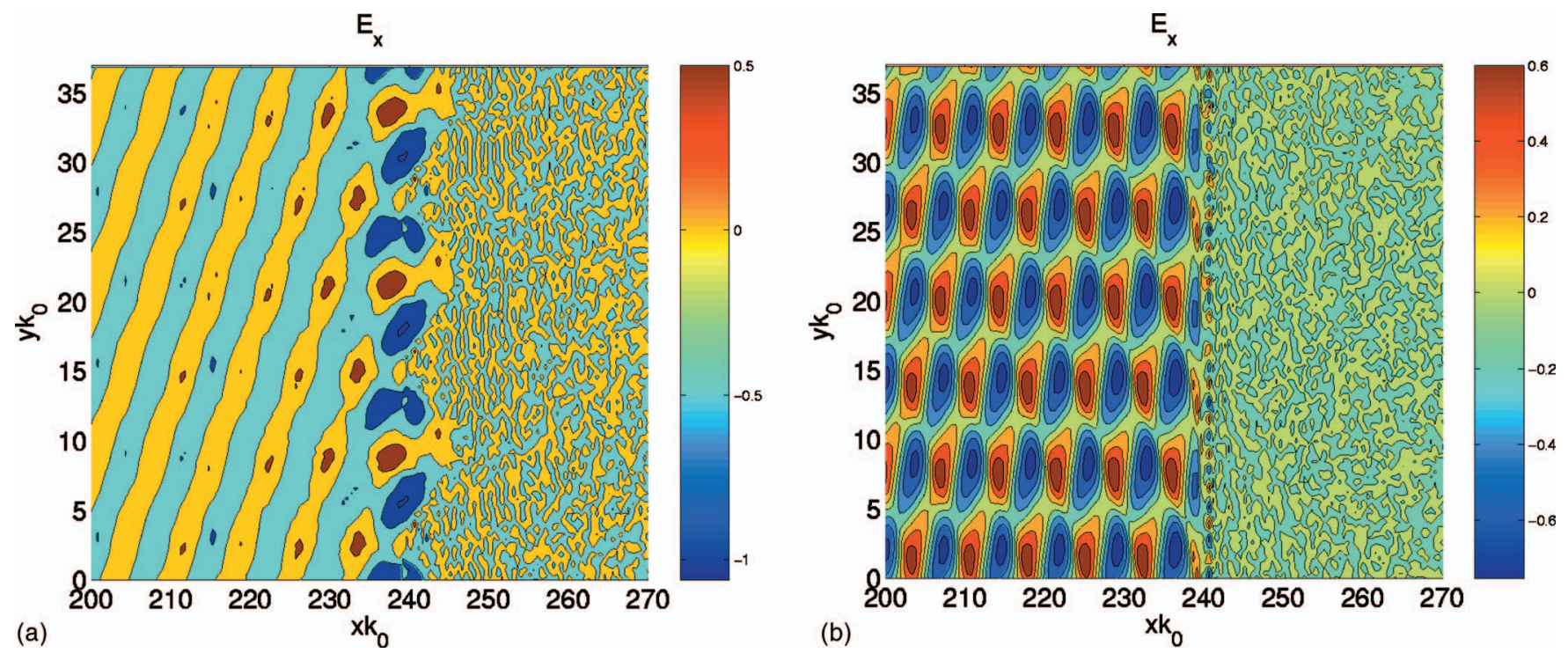

FIG. 1. (Color) Electric field amplitude along the $x$-direction for $I_{0} \lambda_{0}^{2}=10^{18} \mathrm{~W} \mu \mathrm{m}^{2} / \mathrm{cm}^{2}$, pulse duration $60 \mathrm{fs}$, and laser incidence angle of $\sim 30^{\circ}$ at the time when the maximum of the laser pulse is impinging onto the plasma surface located at $x k_{0}=240, t=438 \omega_{0}^{-1}$. Left: case of a modulated plasma surface (resonant case). Right: case of a flat plasma surface (nonresonant case).

$7 \lambda_{0}$ in that direction. Larger plasma sizes, up to $20 \lambda_{0}$, have also been tested but the results did not change significantly. The particle boundary conditions are periodic in the $y$-direction and the fast electrons reaching the right box side are cooled in a buffer. As the skin depth is here equal to $c / \omega_{p e} \sim \lambda_{0} / 30$, we used a grid size of $k_{0} \Delta x=0.05 \sim 4 \lambda_{d}$ and $k_{0} \Delta y=0.036 \sim 3 \lambda_{d}$ with $5900 \times 1020$ grid cells in order to have a correct spatial resolution of a few Debye lengths $\left(\lambda_{d}=v_{\text {the }} / \omega_{p e} \sim \lambda_{0} / 500\right) .100$ particles by cell (total number of particles: $6 \times 10^{8}$ ) were considered and the time step was taken as $\omega_{0} \Delta t=0.035$.

\section{SURFACE PLASMA WAVE EXCITATION}

The physical parameters chosen in our simulations are such that the resonant surface wave at the frequency $\omega_{s w}$ $=\omega_{0}=\omega_{p e} / 5$ is in a regime far from the electrostatic limit defined by $\omega_{s w} \sim \omega_{p e} / \sqrt{2}$. In this situation, the surface wave localized at the plasma-vacuum interface at $x k_{0}=240$, propagates along this surface with a phase velocity $\omega_{s w} / k_{s w, y}$ $\sim 0.98 c$. It deserves to be noticed that relativistic corrections to the momentum of an electron in a relativistic wave will lead to modifications of the dispersion relation (1) (Refs. 18 and 27) together with the resonance conditions for excitation of the surface plasma wave. As a consequence, relativistic detuning will occur, which can induce a saturation of the field amplitude. This detuning has been previously estimated to be less than $10 \%$. $^{19}$

As previously mentioned, surface plasma waves are characterized by a large resonant electric field localized at the plasma-vacuum interface, mainly directed perpendicularly to the surface of the plasma and with a small spatial extension. In order to evaluate the amplitude of the field components, we assumed that the surface plasma wave fields are of the form $E_{y}(x) e^{i\left(\omega_{s w} t-k_{s w, y} y\right)}$, with $E_{y}(x)=\bar{E}_{y}(0)$ $\times\left[\Theta(-x) \exp \left(x / L_{E}\right)+\Theta(x) \exp \left(-x / L_{E p l}\right)\right]$. Here, $\Theta(x)$ denotes the Heaviside step function, $x=0$ corresponds to the position of the plasma-vacuum interface, and $L_{E}$ and $L_{E p l}$ are the evanescence lengths of the surface wave in vacuum and inside the plasma, respectively, given by $L_{E}=1 / \sqrt{k_{y}^{2}-\omega_{s w}^{2} / c^{2}}$ and $L_{E p l}=L_{E} /\left(\omega_{p e}^{2} / \omega_{s w}^{2}-1\right)$ with $L_{E p l} \ll L_{E}{ }^{15}$ In the limit $n_{e} / n_{c}$ $\gg 1$, the field amplitude on the vacuum side is such that $\bar{E}_{x}$ $\sim\left(\omega_{p e} / \omega_{s w}\right) \bar{E}_{y} \gg \bar{E}_{y}$ and is the main component of the resonant electric field.

The amplitude of the total electric field obtained in our PIC simulations includes both the amplitudes of the incident $\left(0.5 \times E_{L}\right.$ at this angle of incidence) and reflected laser fields. When the resonance condition of the surface plasma wave is not satisfied (in the case of a flat surface or when the laser angle of incidence is not $30^{\circ}$ so that the resonant conditions of excitation of the surface plasma waves are not fulfilled) the absorption is weak (see below) and the field feature is the sum of the incident and reflected laser fields. In Fig. 1 (right), the electric field amplitude along the $x$-direction (for $I_{0} \lambda_{0}^{2}=10^{18} \mathrm{~W} \mu \mathrm{m}^{2} / \mathrm{cm}^{2}$ and a pulse duration of $60 \mathrm{fs}$ at the time when the maximum of the laser pulse is impinging onto the plasma surface located at $\left.x k_{0}=240\right)$ is shown in the case of a laser incidence of $30^{\circ}$. If we compute the maximum amplitude of the electric field $E_{x}$ along the $x$-direction as shown in Fig. 2 (right), we find $E_{x} \sim 0.5$ at $x k_{0}=240$ and, at $x k_{0}=200$ (vacuum side), $E_{x} \sim 0.7$ which is about twice $E_{L} \sin \left(30^{\circ}\right)$, that is, the sum of the incident and reflected laser fields. In the case where the resonance conditions are fulfilled, an equivalent plot is shown on Fig. 1 (left). As expected, we observe in this case a rather small field reflection together with an enhancement of the field amplitude at the vacuum-plasma interface at $x k_{0}=240$. This field structure shows, at the vacuum-plasma interface, six periods along the $y$-direction, that is, twice as that of the laser field as is can be seen on the left-hand side of the simulation box. Moreover, this structure of the field located at the plasma-vacuum interface oscillates in time with a period of $2 \pi$. This shows that a surface plasma wave oscillating at a frequency $\omega_{0}$ that satis- 

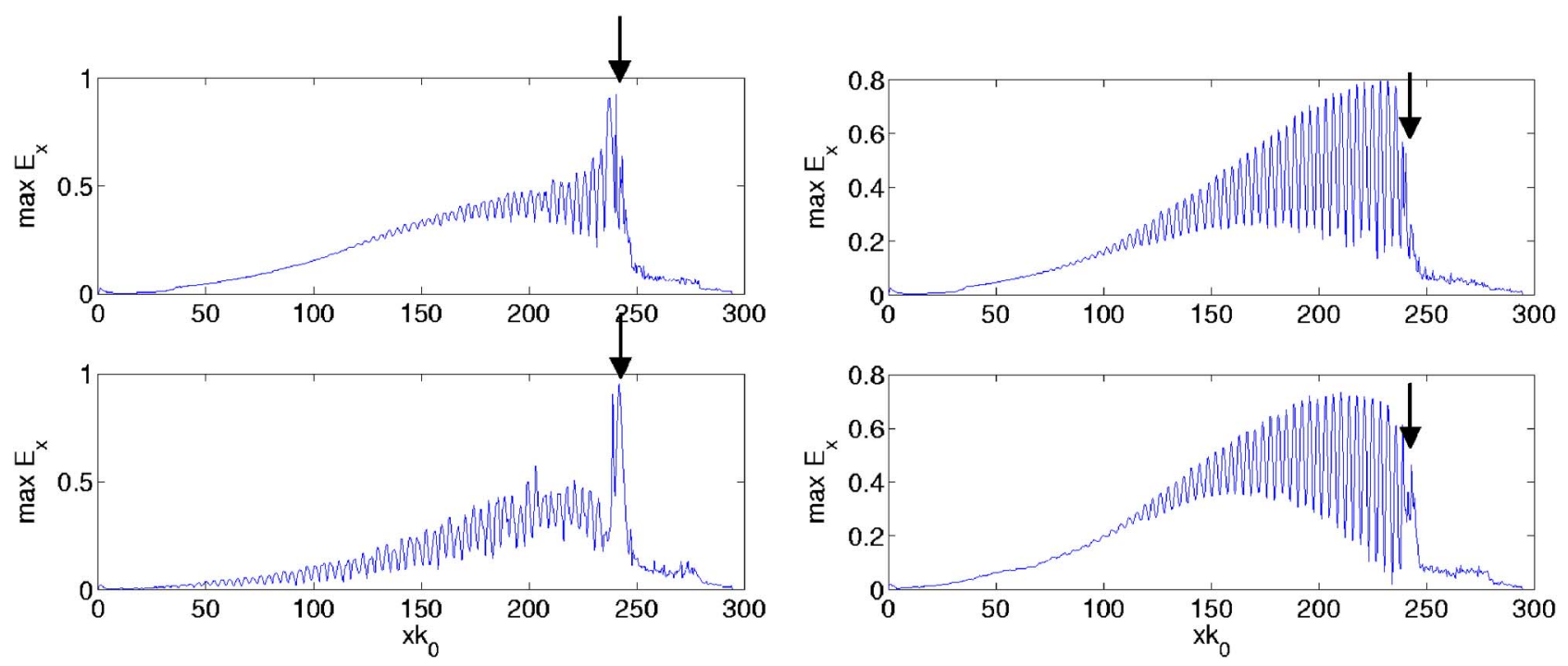

FIG. 2. (Color online) Maximum amplitude of the electric field along the $x$-direction for $I_{0} \lambda_{0}^{2}=10^{18} \mathrm{~W} \mu \mathrm{m}^{2} / \mathrm{cm}^{2}$, laser pulse duration of $60 \mathrm{fs}$ and laser incidence angle of $\sim 30^{\circ}$. The arrows represent the position of the plasma surface at $x k_{0}=240$. Left: case of a modulated plasma surface (resonant case). Right: case of a plane plasma surface (nonresonant case). Up: results when the maximum of the laser pulse is impinging the plasma surface at $t=438 \omega_{0}^{-1}$. Down: results at the same time plus $1 / 3$ of the pulse duration at $t=531 \omega_{0}^{-1}$.

fies the surface plasma wave dispersion relation has been excited here. If we consider the maximum amplitude of the electric field $E_{x}$ along the $x$-direction as shown in Fig. 2 (left), we find $E_{x} \sim 0.9$ at $x k_{0}=240$ and, at $x k_{0}=200, E_{x}$ $\sim 0.4$ which gives an enhancement of the field amplitude onto the plasma surface located at $x k_{0}=240$ by a factor $\eta$ $=E_{x} / E_{L}$ of about 2 . This enhancement become larger at a later time, as the laser field amplitude diminishes while the surface plasma wave amplitude stays at roughly the same value until the main of the laser pulse at time $t \sim 560 \omega_{0}^{-1}$ has reached the plasma surface, after which it gradually damps. The maximum value reached is $\eta \sim 4$.

Actually, the relatively low value of the instantaneous amplification factor $\eta$ obtained here for the surface plasma wave results from a saturation effect of the field amplitude due to the very efficient particle heating mechanism (as it will be seen below), which corresponds to a strong damping of the surface plasma wave. Inspection of the density profile and comparison with motionless ion simulations in the same conditions indicate that, in the time scale considered here, a possible saturation mechanism for the surface plasma wave through any modification of the density profile due to the hydrodynamic expansion of the plasma toward the vacuum can be ruled out. In the same way, relativistic detuning, as mentioned above, cannot explain such a low amplification factor. $^{18,19,27}$ In fact, we can infer that the wave damping is mainly due to the strong acceleration of the electrons in the field of the surface plasma wave. This statement can be verified by calculating the total energy acquired by those electrons that have left the surface inside and outside the plasma at maximum amplitude of the laser pulse that occurs at time $t=438 \omega_{0}^{-1}$. The average kinetic energy per particle is approximately given by $\langle\mathcal{E}\rangle \sim 0.01 m_{e} c^{2}$. Thus, the total kinetic energy is $\mathcal{E}_{\text {Tot }} \sim\langle\mathcal{E}\rangle n_{e} S L_{\mathrm{pl}}$, where $S$ is the transverse size of the system, given by the size of the focal laser spot, and where we can take $L_{\mathrm{pl}} \sim 6 \lambda_{0}$ in order to take into account the fact that we have excluded the particles at the surface. Now, if we compare this quantity with the surface wave energy approximately given by $\left(E_{s w}^{2} / 4 \pi\right) S L_{E}$, considering the value of $E_{s w}$ at the maximum of the pulse, we find that the average total electron kinetic energy is just of the same order as the energy of the surface plasma wave field, which corresponds to a very strong damping rate comparable to that resulting from the laser pulse envelope.

\section{ELECTRON ACCELERATION BY SURFACE PLASMA WAVES}

As previously inferred, ${ }^{19}$ the existence of a strong inhomogeneous high-frequency electric field at the vacuumplasma interface should strongly affect the electron behavior near the plasma surface. To single out the role of the surface plasma wave, we first compare the electron kinetic energy distribution function in the presence or in the absence of surface plasma wave, as plotted in Fig. 3. The distribution function is averaged over the whole space, which includes those electrons that have been accelerated outside and inside the plasma. The part played by the resonantly excited surface plasma wave is clearly visible as the maximum energy acquired by the electrons at the peak of the laser pulse is three times larger than in the case where the surface plasma wave is not excited. Furthermore, it can be seen that a much larger number of electrons are accelerated to high energies. The surface wave acts as a resonator, highly increasing the efficiency of the electron acceleration mechanism, with most of the laser energy being stored by the surface wave which then dissipates into the plasma. Thus, electrons are more efficiently accelerated in the presence of a surface plasma wave excitation and a much larger percentage of the laser energy is absorbed by the plasma. This fact is clearly evidenced by considering the laser absorption over the whole simulation time; in the presence of the surface plasma wave, we find an 


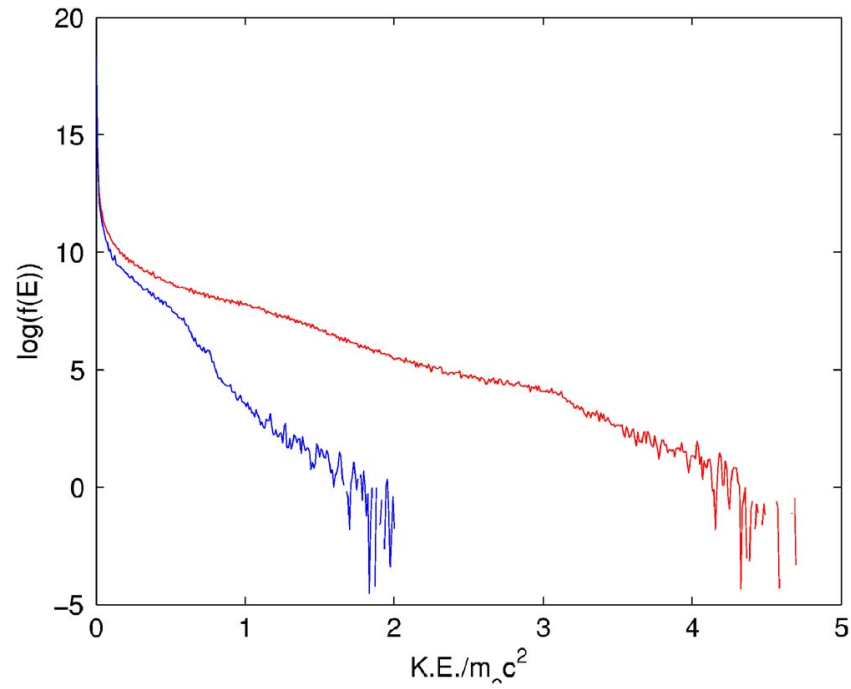

FIG. 3. (Color online) Decimal logarithm of the average electron energy distribution function at $t=438 \omega_{0}^{-1}$ (peak time of the laser pulse). In red: curve corresponding to the case where the plasma surface is modulated (resonant case). In blue: results for the case of a flat plasma surface (nonresonant case).

absorption of $68 \%$, a value to set against the only $18 \%$ obtained in the absence of a surface wave. This dramatically enhanced absorption obtained in the presence of a surface plasma wave is higher than that obtained at higher laser intensity and in normal incidence in the case of an induced rippling of the plasma surface. ${ }^{28}$

It is also interesting to compare the maximum reached electron kinetic energy $\mathcal{E}_{\mathrm{Max}}$ with the ponderomotive energy that can be gained due to the component of the surface wave field perpendicular to the surface (to wit, the largest component of the surface plasma wave). In the relativistic limit, this energy corresponds to a maximum given by

$$
\mathcal{E}_{\mathrm{Max}} \sim m_{e} c^{2}\left(\gamma_{\mathrm{osc}}-1\right) \quad \text { with } \gamma_{\mathrm{osc}}=\left(1+p_{\mathrm{osc}}^{2} / m_{e}^{2} c^{2}\right)^{1 / 2}
$$

and

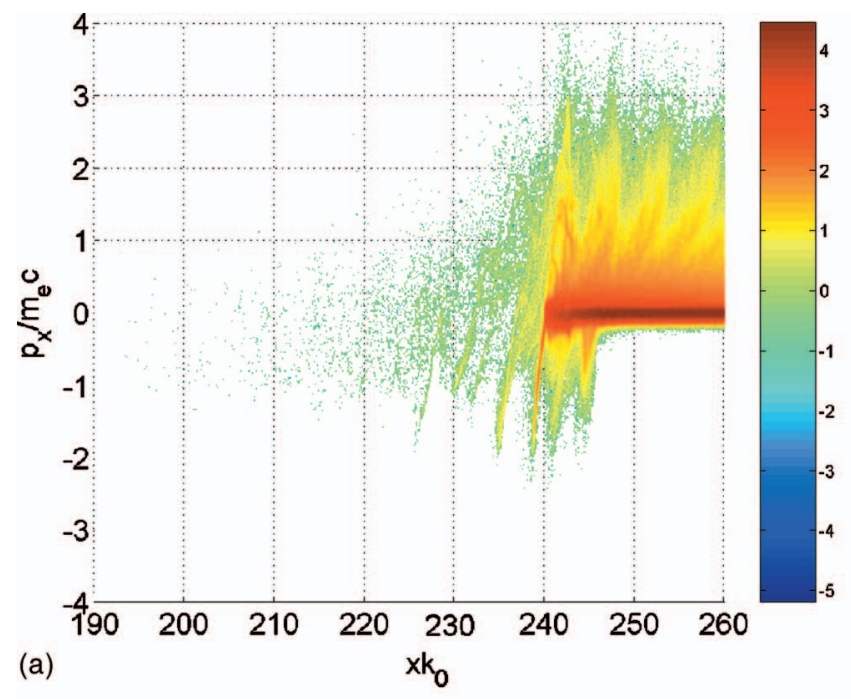

$$
\begin{aligned}
p_{\mathrm{osc}} / m_{e} c= & e E_{s w} /\left(m_{e} c\right) \omega_{0}=8.6 \\
& \times 10^{-10}\left[\eta^{2} I_{0}\left(\mathrm{~W} / \mathrm{cm}^{2}\right) \lambda_{0}^{2}\left(\mu \mathrm{m}^{2}\right)\right]^{1 / 2}
\end{aligned}
$$

Hence, taking an average value for the field enhancement factor such that $\eta^{2} \sim 10$ and $I_{0} \lambda_{0}^{2}=10^{18} \mathrm{~W} \mu \mathrm{m}^{2} / \mathrm{cm}^{2}$, we find that $\gamma_{\text {osc }}=2.89$ and $\mathcal{E}_{\mathrm{Max}} \sim 2 m_{e} c^{2} \sim 1 \mathrm{MeV}$. For comparison, simply taking $\eta=1$, one would get $\gamma_{\text {osc }}=1.32$ and $\mathcal{E}$ $\sim 0.32 m_{e} c^{2} \sim 0.16 \mathrm{MeV}$. These values are significantly smaller than those obtained in our simulations (see Fig. 3) where the electrons are accelerated up to an energy value of $4.5 m_{e} c^{2} \sim 2.3 \mathrm{MeV}$.

Moreover, as expected from previous studies, ${ }^{19}$ pulsed bunches of hot electrons at frequency $\omega_{0}$ are clearly apparent in Fig. 4 (left) along the $x$-direction perpendicular to the vacuum-plasma interface inside the plasma by inspecting the electron phase space $\left(p_{x} / m_{e} c, x k_{0}\right)$ (see Ref. 29). In the vacuum where the packets of accelerated electrons are mixed with those pulled back to the plasma by the space charge field, the bunches of electrons are less visible at this moment of time. Now, in the absence of excited surface waves, the acceleration along the $x$-direction is clearly weaker and essentially due to the component of the laser field perpendicular to the surface (which also contributes to laser energy absorption through the Brunel effect) and to the ponderomotive force resulting from the $\vec{J} \times \vec{B}$ term of the Lorentz force. Hence, the electrons are less accelerated by the two oscillating forces at frequencies $\omega_{0}$ and $2 \omega_{0}$. As the amplitudes of those forces are significantly smaller than that due to the field of the surface plasma wave, the phase space is smoother in comparison with the case where the surface plasma wave is excited, as it is clearly exhibited in Fig. 4.

To complete the analysis, we have to take into account the component of the field of the surface plasma wave propagating along the surface which also contributes to transfer to the electrons some momentum along the surface in the negative $y$-direction. This effect is clearly apparent through inspection of the $\left(p_{y} / m_{e} c, x k_{0}\right)$ phase space shown in Fig. 5, where one can perceive a large heating in the negative $p_{y}$

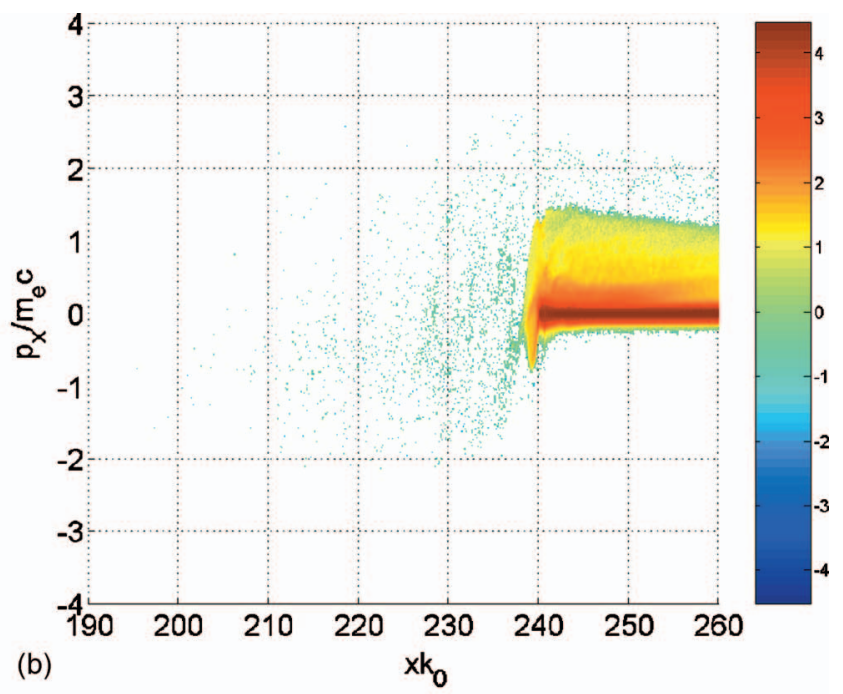

FIG. 4. (Color) Electron phase space $p_{x} / m_{e} c$ versus $x k_{0}$ at $t=438 \omega_{0}^{-1}$ (peak time of the laser pulse) for the resonant (left) and nonresonant (right) cases. 

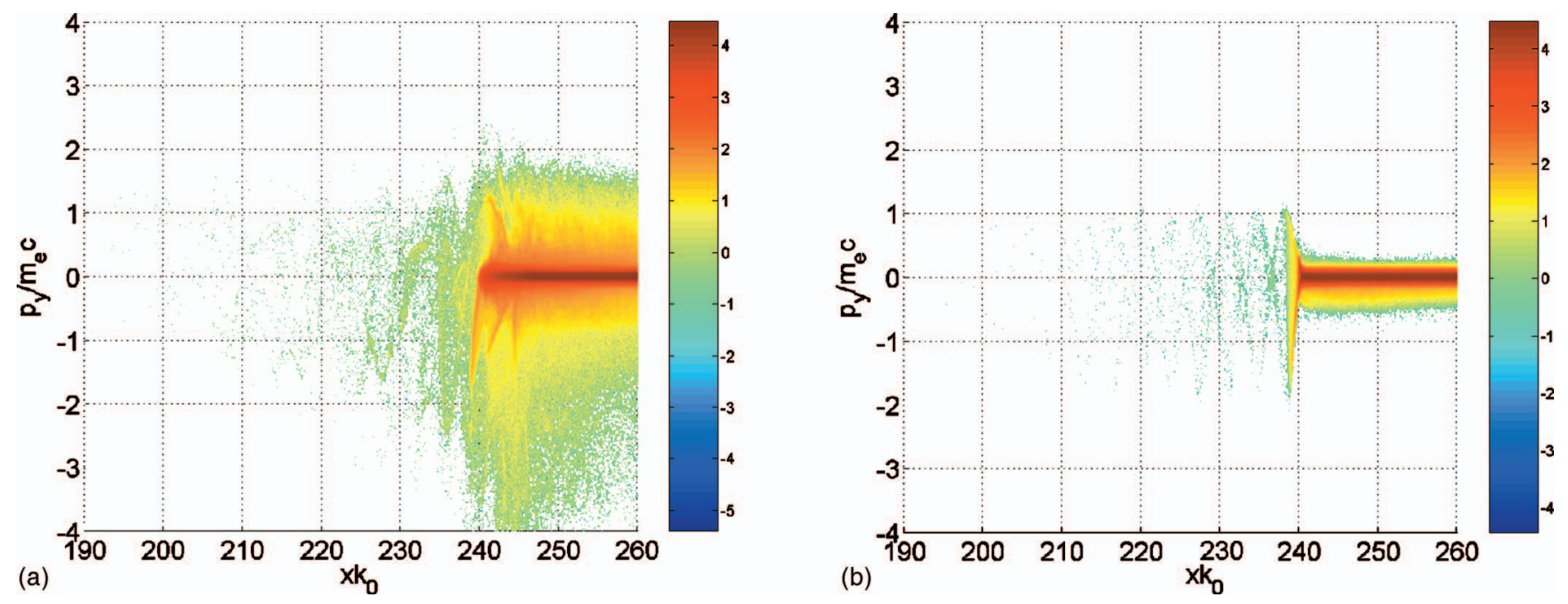

FIG. 5. (Color) same as in Fig. 4 but for $p_{y} / m_{e} c$.

direction. In the same figure, one can also observe packets of electrons heated symmetrically in both positive and negative $p_{y}$ directions. To interpret this result, one can notice that the phase space, although more diffuse in our case, is very similar to what has been already observed ${ }^{30,31}$ at higher laser intensities and denser plasmas due to the self-generation of large quasistatic magnetic fields ${ }^{32-34}$ at the plasma surface. In our case, the magnetic field component at the surface is dominated by the resonant oscillating magnetic field of the surface wave. However, we also observe very early (at time $t \sim 350 \omega_{0}^{-1}$ ) in our simulations low-frequency (quasistatic) self-generated magnetic fields, clearly evident close to the top of the plasma modulation. The spatial extension of the magnetic fields is of the order of $\lambda_{0} / 2$ and their amplitude grows in time to reach a maximum of roughly $20 \mathrm{MG}$.

Furthermore, the large heating in the negative $y$-direction could be understood as due to wavebreaking of the surface plasma wave. Although generally smaller than the $E_{x}$ component, the $E_{y}$ component of the surface plasma wave is to be accounted for as, in the present case, its amplitude is approximately half of the laser field amplitude. To perceive more explicitly the part played in our simulations by the wave breaking process, let us first consider the hypothesis of a cold plasma. In this case, the wave breaking condition is given by $v_{y} \geqslant v_{\mathrm{ph}}$, where $v_{\mathrm{ph}}=\omega_{s w} / k_{s w, y} \sim c$ and $v_{y}=\sqrt{2 e} \Phi / m_{e}$, with $E_{y}=-\nabla \Phi \sim k_{s w, y} \Phi$. This condition can be rewritten in the form, $\left(2 e E_{y}\right) /\left(c \omega_{s w} m_{e}\right) \geqslant 1$. As for the aboveconsidered laser incidence angle $E_{y} \sim 0.5 E_{L}$, we obtain $\left(2 e E_{y}\right) /\left(c \omega_{s w} m_{e}\right) \sim 0.85$, so that the wave breaking condition is not satisfied in the cold plasma hypothesis. However, when the peak of the laser pulse has reached the plasma surface, the initially cold plasma has already been substantially heated, up to relativistic velocities. Thus, we are actually dealing with electron populations in the plasma that are characterized by strongly different temperatures. Indeed, this heating effect can lead to breaking of surface plasma wave. For a hot plasma, the wave breaking condition ${ }^{35}$ is then $\left(2 e E_{y}\right) /\left(c \omega_{s w} m_{e}\right) \geqslant\left[(4 / 27)\left(m_{e} c^{2} / T_{e}\right)\right]^{1 / 4}$. This last term becomes equal to 0.52 for an electron temperature correspond- ing to $T_{e}=2 m_{e} c^{2}$, as shown in Fig. 3, so that the wave breaking condition is fulfilled. Wave breaking can thus explain the large particle acceleration in the negative $y$-direction at this moment of time. Therefore, two-dimensional effects together with the contribution of the self-generated low-frequency magnetic fields act favorably on the system by increasing the average particle heating. It is worth noting that when the surface plasma wave does not exist, opposite results are found for particle heating through the Brunel mechanism ${ }^{12}$ where two-dimensional effects tend to decrease its efficiency and to saturate the absorption at a lower level.

Lately, much attention has been given to the angular distribution of hot electrons as it is relevant for the potential applications of electron and proton acceleration. ${ }^{37-40}$ It is thus of interest, for comparison with experimental results, to calculate the electron energy distribution function for different angles with respect to the vacuum-plasma interface. In Fig. 6, we have reported the logarithm of the electron distribution function as a function of angle and energy in the case of a flat plasma surface. The fastest electrons are mainly emitted at $-20^{\circ}$ inside the plasma and at $-160^{\circ}$ in the vacuum (the angle $\vartheta=0^{\circ}$ corresponding to particles propa-

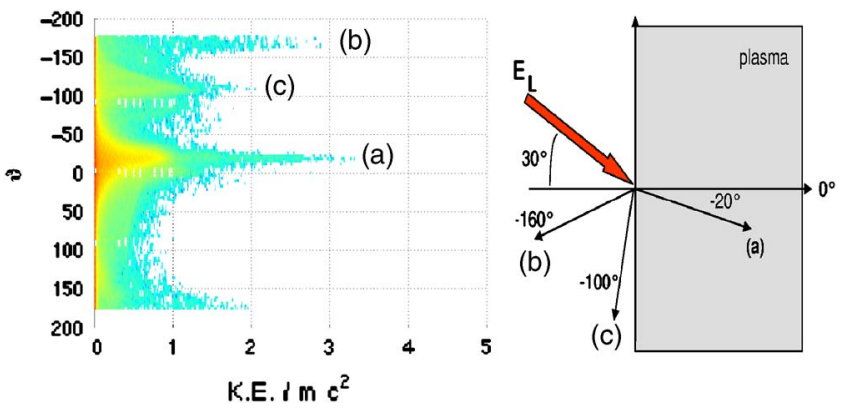

FIG. 6. (Color online) Nonresonant case: Electron distribution function at $t=531 \omega_{0}^{-1}$ (peak time $+1 / 3$ of the laser pulse duration) as a function of electron energy and direction of propagation. $\vartheta=0$ corresponds to electrons that are moving perpendicularly to the plasma vacuum interface inside the plasma, as represented on the right-hand side. 


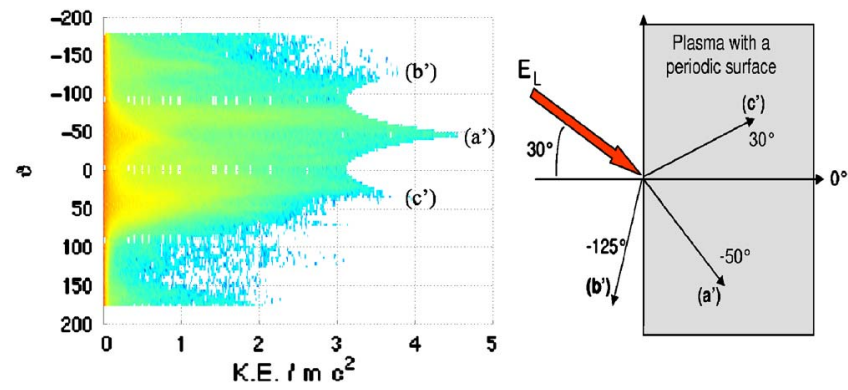

FIG. 7. (Color online) Same as in Fig. 6 but in the resonant case.

gating inside the plasma along the normal to the surface). Those values, close to the specular direction of the laser, are in agreement with previous theoretical and numerical predictions for electron acceleration by laser in weakly overdense flat plasmas. ${ }^{37}$ In addition, it is to be noticed that, due to the high value of $n_{e} / n_{c}$, the electron surface current is clearly apparent as another peak of emission at $\vartheta=-90^{\circ}$. By contrast, in the presence of the excited surface plasma wave, it can be seen in Fig. 7 that the main directions of electron emission are modified: the electrons with the largest energy are emitted at $-50^{\circ}$ inside the plasma. Many fast electrons are also emitted at $+30^{\circ}$ inside the plasma, and at $-125^{\circ}$ in the vacuum. This behavior is related to the existence of the self-generated magnetic fields which transfer momentum to the electrons near the surface.

In both cases, more electrons are accelerated inside the plasma than outside, an effect that is greatly enhanced in the presence of the surface plasma wave. This result can be interpreted as due to the strong surface charge field created by the electrons leaving the plasma toward the vacuum. This space charge field thus leads to a sort of "enhanced vacuum heating" in the presence of the surface plasma wave. Note that in these figures, the quiver motion of the particles close to the surface in the laser field and in the surface wave field is included but represents a negligible contribution to the distribution function at this moment of time.

\section{ION ACCELERATION}

At the end of the interaction in the short pulse regime considered here, strong electron acceleration can result in efficient ion acceleration provided that the condition of detachment of an electron bunch from the plasma is not satisfied. ${ }^{36}$ This will occur if the number $N_{b}$ of accelerated electrons in the bunch of diameter $d$ is too large for a given electron energy, that is, if $\gamma_{b}-1<N_{b} e^{2} /\left(m_{e} c^{2} d\right)$, where $\left(\gamma_{b}\right.$ $-1) m_{e} c^{2}$ is the relativistic kinetic energy of the electrons. The bunch length is considered here much smaller than its diameter. In the case of an highly overdense plasma, even for relativistic values of $\gamma_{b} \sim 10$, this condition is typically satisfied except for a small population of very energetic electrons that will possibly escape from the plasma. We have seen in the preceding section that the excitation of a surface plasma wave greatly increases the formation of relativistic electron bunches, for a laser intensity as low as $I_{0} \lambda_{0}^{2}$ $=10^{18} \mathrm{~W} \mu \mathrm{m}^{2} / \mathrm{cm}^{2}$. It is then of interest to look at the ion behavior in longer time scales in the framework of short laser pulse interaction.

In Figs. 8 and 9, we have plotted the ion phase space $p_{x} / m_{e} c$ versus $x k_{0}$ and $p_{y} / m_{e} c$ versus $x k_{0}$ at $t=897 \omega_{0}^{-1}$ that corresponds to a time when the short-pulse laser light is not impinging any more onto the surface of the plasma while the surface plasma wave is almost completely damped. It can clearly be observed that the acceleration of ions toward the vacuum is much more efficient when the surface plasma wave is excited. Actually, this effect is a consequence of the fact that the plasma expansion is here essentially governed by the space charge fields, the importance of which is as much greater as the effective temperature of the electrons into the vacuum increases. ${ }^{41}$ Moreover, in both cases, the simulations show compression of the plasma surface along with ion acceleration inside the plasma (see Figs. 8 and 9) due to the light pressure as soon as the peak of the laser pulse has reached the plasma surface. This compression effect appears as a sharp shoulder of the ion density at the surface
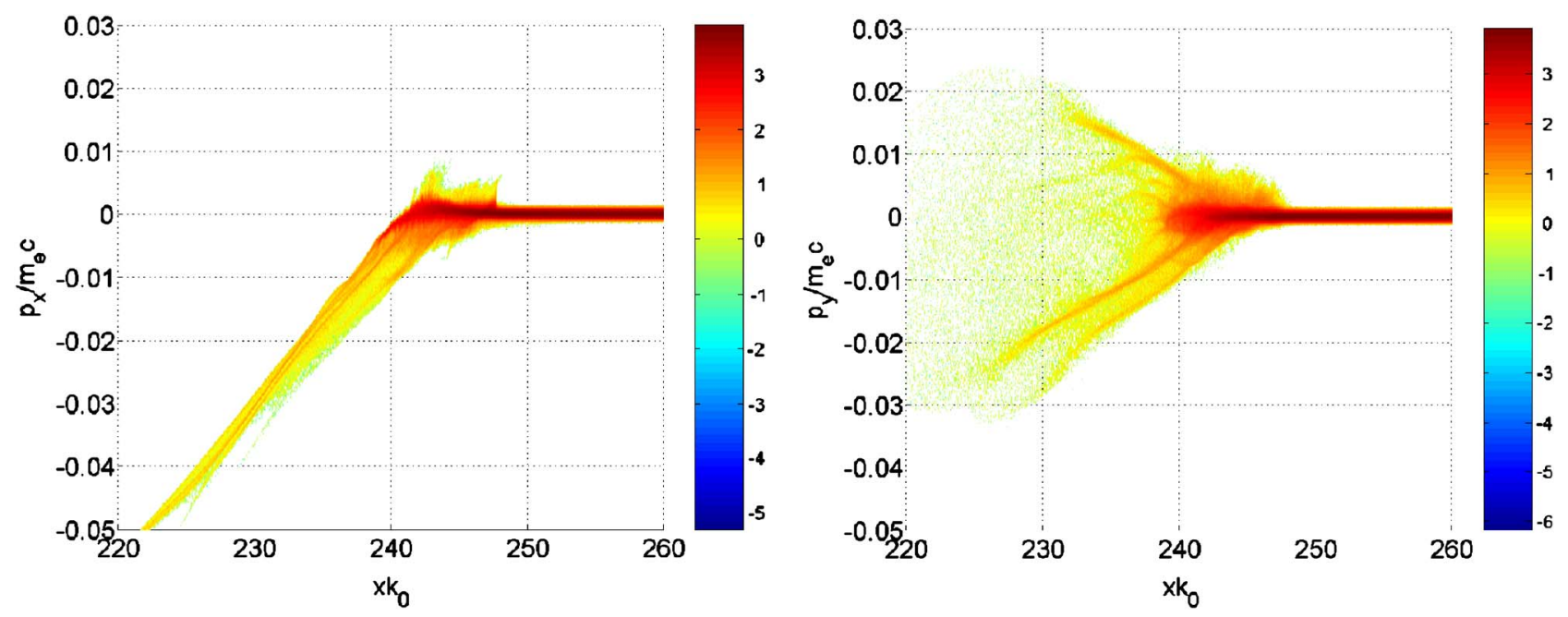

FIG. 8. (Color online) Ion phase space $p_{x} / m_{e} c$ versus $x k_{0}$ at $t=897 \omega_{0}^{-1}$ (left) and $p_{y} / m_{e} c$ versus $x k_{0}$ (right) in the resonant case. 

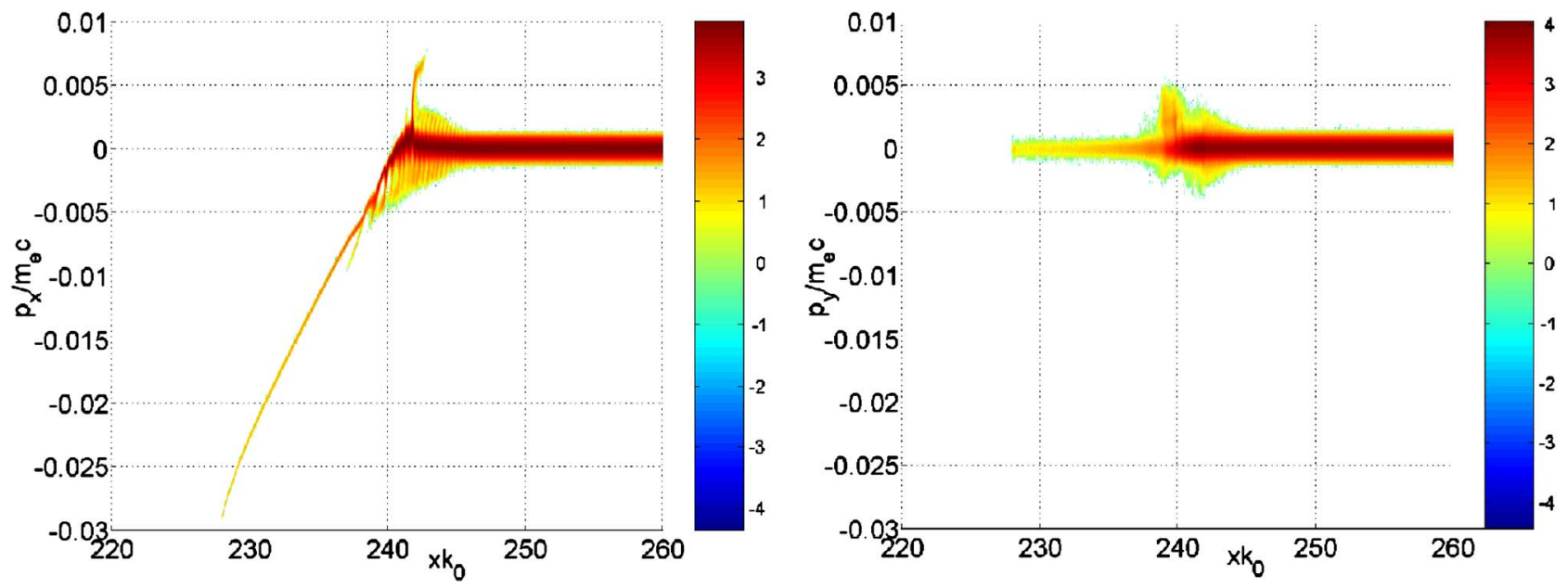

FIG. 9. (Color online) Same as in Fig. 8 but in the nonresonant case.

with a density increment that can reach up to $50 \%$, as observed in density snapshots (not shown here).

For longer laser pulse duration, additional interaction processes such as resonant absorption can take place as the density profile of the plasma strongly evolves with time. However, up to $250 \mathrm{fs}$, our test simulations show that the surface plasma wave together with the enhanced laser absorption and electron acceleration described here are still observed. The above-mentioned additional processes that contribute also to ion acceleration through generation of suprathermal electrons could then be affected by the excitation of a surface plasma wave. Thus, conversion of the surface wave energy could contribute to improve the efficiency of ion acceleration mechanisms in laser pulse regimes longer than some tens of femtoseconds but shorter than a picosecond for which the plasma surface could support instabilities which prevent surface wave excitation. This question which would require a more detailed analysis of the ion dynamics over longer time scales will be the subject of further investigations.

\section{CONCLUSION}

In this paper, we have presented the results of numerical investigations by means of a 2D PIC code about resonant excitation of surface plasma waves by ultrashort $(<100 \mathrm{fs})$ high-intensity laser pulses $\left(I_{0} \lambda_{0}^{2}=10^{18} \mathrm{~W} \mu \mathrm{m}^{2} / \mathrm{cm}^{2}\right)$ in overdense plasmas $\left(n_{e} / n_{c}=25\right)$. A remarkable result is a dramatic increase of light absorption by the plasma from $18 \%$ to $68 \%$. Another significant result is the creation of a relatively large population of highly energetic electrons $(2.3 \mathrm{MeV})$. It is worth noting that such high electron energies cannot be explained by standard laser ponderomotive effects, nor $\vec{J} \times \vec{B}$ mechanism, nor vacuum heating. Thus, resonant excitation of surface plasma waves appears as a highly performing method to reach relativistic electron energies for the aboveconsidered laser intensities. Detailed analysis of the particle phase space have revealed the leading part played by the transverse dynamics when the surface plasma wave is excited. We have also shown that the ion motion toward the vacuum is drastically affected by the high electron heating.
Therefore, advantage could be taken of the enhanced energy absorption through the excitation of surface plasma waves to improve the efficiency of proton acceleration mechanisms in the framework of short laser pulse interaction $(<500 \mathrm{fs})$. This general picture has been confirmed by further simulations at higher laser intensities (up to $I_{0} \lambda_{0}^{2}=10^{19}$ $\mathrm{W} \mu \mathrm{m}^{2} / \mathrm{cm}^{2}$ ). Hence, an even better value of the absorption rate along with production of still higher electron bunch energies can be expected.

${ }^{1}$ S. J. Gitomer, R. D. Jones, F. Begay, A. W. Ehler, J. F. Kephart, and R. Kristal, Phys. Fluids 29, 2679 (1986); J. D. Crow, P. L. Auer, and J. E. Allen, J. Plasma Phys. 14, 65 (1975); J. Fuchs, T. E. Cowan, P. Audebert et al., Phys. Rev. Lett. 91, 255002 (2003); L. Disdier, J. P. Garonnet, G. Malka, and J. L. Miquel, ibid. 82, 1454 (1999).

${ }^{2}$ Y. Sentoku, K. Mima, S. Miyamoto, and Y. Kishimoto, Phys. Plasmas 5, 4366 (1998); K. B. Wharton, S. P. Hatchett, S. C. Wilks, M. H. Key, J. D. Moody, V. Yanovsky, B. A. Hammel, M. D. Perry, and C. Joshi, Phys. Rev. Lett. 81, 822 (1998); S. Bastiani, A. Rousse, J. P. Geindre, P. Audebert, C. Quoix, G. Hamoniaux, A. Antonetti, and J. C. Gauthier, Phys. Rev. E 56, 7179 (1997); P. A. Norreys, M. Santala, E. Clark et al., Phys. Plasmas 6, 2150 (1999).

${ }^{3}$ M. Tabak, J. Hammer, M. E. Glinsky, W. L. Kruer, S. C. Wilks, J. Woodworth, M. Campbell, M. D. Perry, and R. J. Mason, Phys. Plasmas 1, 1626 (1994).

${ }^{4}$ P. J. Channell, Laser Acceleration of Particles (AIP, New York, 1982), and references therein; E. Esarey, P. Sprangle, J. Krall, and A. Ting, IEEE Trans. Plasma Sci. 24, 252 (1996).

${ }^{5}$ C. Joshi and T. C. Katsouleas, Laser Acceleration of Particles (AIP, New York, 1985).

${ }^{6}$ T. Tajima and J. M. Dawson, Phys. Rev. Lett. 43, 267 (1979); C. Joshi, W. B. Mori, T. Katsouleas, J. M. Dawson, J. M. Kindel, and D. W. Forslund, Nature (London) 311, 525 (1984); T. C. Chiou, T. Katsouleas, C. Decker, W. B. Mori, J. S. Wurtele, G. Shvets, and J. J. Su, Phys. Plasmas 2, 310 (1995); D. Umstadter, J. K. Kim, and E. Dodd, Phys. Rev. Lett. 76, 2073 (1996); E. Esarey, P. Sprangle, J. Krall, and A. Ting, ibid. 79, 2682 (1997); V. Yakimenko, I. V. Pogorelsky, I. V. Pavlishin et al., ibid. 91, 014802 (2003); N. Saito and A. Ogata, Phys. Plasmas 10, 3358 (2003); L. M. Gorbunov, P. Mora, and R. R. Ramazashvili, ibid. 10, 4563 (2003).

${ }^{7}$ H. M. Milchberg, R. R. Freeman, S. C. Davey, and R. M. More, Phys. Rev. Lett. 61, 2364 (1988); M. M. Murnane, M. C. Kapteyn, and R. W. Falcone, ibid. 62, 155 (1989); J. A. Cobble, G. A. Kyrala, A. A. Hauer, A. J. Taylor, C. C. Gomez, N. D. Delamater, and G. T. Schappert, Phys. Rev. A 39, 454 (1989); J. C. Gauthier, J. P. Geindre, P. Audebert, S. Bastiani, C. Quoix, G. Grillon, A. Mysyrowicz, A. Antonetti, and R. C. Mancini, Phys. Plasmas 4, 1811 (1997); K. Eidmann, R. Rix, T. Schlegel, and K. Witte, Europhys. Lett. 55, 334 (2001).

${ }^{8}$ M. I. K. Santala, M. Zepf, I. Watts et al., Phys. Rev. Lett. 84, 1459 (2000); 
X. Wang, M. Krishnan, N. Saleh, H. Wang, and D. Umstadter, ibid. 84, 5324 (2000); W. Yu, V. Bychenkov, Y. Sentoku, M. Y. Yu, Z. M. Sheng, and K. Mima, ibid. 85, 570 (2000); Q. L. Dong and J. Zhang, Phys. Plasmas 8, 1025 (2001).

${ }^{9}$ J. Kupersztych and M. Raynaud, Phys. Rev. E 59, 4559 (1999).

${ }^{10}$ P. J. Catto and R. M. More, Phys. Fluids 20, 704 (1977).

${ }^{11}$ W. Kruer and K. Estabrook, Phys. Fluids 28, 430 (1985).

${ }^{12}$ F. Brunel, Phys. Rev. Lett. 59, 52 (1987); Phys. Fluids 31, 2714 (1988).

${ }^{13}$ S. Ichimaru, Basic Principles of Plasma Physics (Benjamin, Reading, 1973).

${ }^{14}$ H. Cai, W. Yu, S. Zhu, C. Zheng, L. Cao, B. Li, Z. Y. Chen, and A. Bogerts, Phys. Plasmas 13, 094504 (2006).

${ }^{15}$ P. K. Kaw and J. B. McBride, Phys. Fluids 13, 1784 (1970).

${ }^{16}$ J. M. Kindel, K. Lee, and E. L. Lindman, Phys. Rev. Lett. 34, 134 (1975).

${ }^{17}$ R. Dragila and P. Vukovic, Phys. Rev. Lett. 61, 2759 (1988).

${ }^{18}$ A. Macchi, F. Cornolti, F. Pegoraro, T. V. Liseikina, H. Ruhl, and V. A. Vshivkov, Phys. Rev. Lett. 87, 205004 (2001).

${ }^{19}$ J. Kupersztych, M. Raynaud, and C. Riconda, Phys. Plasmas 11, 1669 (2004).

${ }^{20}$ Y. P. Bliokh, J. Felsteiner, and Y. Slutsker, Phys. Rev. Lett. 95, 165003 (2005).

${ }^{21}$ J. C. Gauthier, A. Rousse, F. Fallies, J. P. Geindre, P. Audebert, A. Mysyrowicz, G. Grillon, and J. P. Chambaret, Proc. SPIE 2523, 242 (1995).

${ }^{22}$ J. Kupersztych, P. Monchicourt, and M. Raynaud, Phys. Rev. Lett. 86, 5180 (2001).

${ }^{23}$ S. E. Irvine, A. Dechant, and A. Y. Elezzabi, Phys. Rev. Lett. 93, 184801 (2004); S. E. Irvine and A. Y. Elezzabi, Appl. Phys. Lett. 86, 264102 (2005).

${ }^{24}$ J. Zawadzka, D. A. Jaroszynski, J. J. Carey, and K. Wynne, Nucl. Instrum. Methods Phys. Res. A 445, 324 (2000); Appl. Phys. Lett. 79, 2130 (2001).

${ }^{25}$ Developed at CPhT, Ecole Polytechnique.
${ }^{26}$ H. Raether, Surface Plasmons on Smooth and Rough Surfaces and Gratings (Springer-Verlag, Berlin, 1988).

${ }^{27}$ A. I. Akhiezer and R. V. Polovin, Sov. Phys. JETP 5, 696 (1956).

${ }^{28}$ C. Ren, M. Tzoufras et al., Phys. Plasmas 13, 056308 (2006), and references therein.

${ }^{29}$ The phase space output shown in Figs. 4, 8-left and 9-left (respectively, Figs. 5, 8-right and 9-right) is a normalized distribution function $f\left(x, y, p_{x}, p_{y}\right)$, subsequently averaged over $p_{y}$ and $y$ (respectively, $p_{x}$ and $y$ ). This procedure of averaging over $y$, because of the distribution inhomogeneity, affects the weight associated to a given $\left(p_{y}, p_{x}\right)$, all the values of $\left(p_{y}, p_{x}\right)$ being nevertheless included. In the isocontour log plot reported here the effect of the averaging is smoothed out.

${ }^{30}$ H. Popescu, S. D. Baton F. Amiranoff et al., Phys. Plasmas 12, 063106 (2005).

${ }^{31}$ H. Ruhl, Y. Sentoku, K. Mima, K. A. Tanaka, and R. Kodama, Phys. Rev. Lett. 82, 2095 (1999).

${ }^{32}$ T. Nakamura, S. Kato, H. Nagamoto, and K. Mima, Phys. Rev. Lett. 93, 265002 (2004)

${ }^{33}$ J. C. Adam, A. Heron, and G. Laval, Phys. Rev. Lett. 97, 205006 (2006).

${ }^{34}$ M. Lazar, R. Schlickeiser, and P. K. Shukla, Phys. Plasmas 13, 102302 (2006).

${ }^{35}$ T. P. Coffey, Phys. Fluids 7, 1402 (1971); R. M. G. M. Trines, and P. A. Norreys, Phys. Plasmas 13, 123102 (2006), and references therein.

${ }^{36}$ V. T. Tikhonchuk, Phys. Plasmas 9, 1416 (2002).

${ }^{37}$ M. Chen, Z. M. Sheng, and J. Zhang, Phys. Plasmas 13, 014504 (2006); Z. M. Sheng, Y. Sentoku, K. Mima, J. Zhang, W. Yu, and J. Meyer-terVehn, Phys. Rev. Lett. 85, 5340 (2000).

${ }^{38}$ Z. Li, H. Daido, A. Fukumi et al., Phys. Plasmas 13, 043104 (2006).

${ }^{39}$ Y. T. Li, X. H. Yuan, M. H. Xu et al., Phys. Rev. Lett. 96, 165003 (2006).

${ }^{40}$ H. Habara, K. Adumi, T. Yabuuchi et al., Phys. Rev. Lett. 97, 095004 (2006).

${ }^{41}$ P. Mora, Phys. Rev. Lett. 90, 185002 (2003), and references therein. 